\title{
Author's reply: Measles on a cruise ship - links with the outbreak in the Philippines
}

S Lanini1, M R Capobianchi (maria.capobianchi@inmi.it) ${ }^{1}$, T Derrough ${ }^{2}$, E Severi² ${ }^{2}$ L Vellucci ${ }^{3}$, M G Pompa $^{3}$

1. National Institute for Infectious Diseases (INMI) "Lazzaro Spallanzani” Rome, Italy

2. European Centre for Disease Prevention and Control (ECDC), Stockholm, Sweden

3. Ministry of Health, Directorate General for Prevention, Rome, Italy

Citation style for this article:

Lanini S, Capobianchi MR, Derrough T, Severi E, Vellucci L, Pompa MG. Author's reply: Measles on a cruise ship - links with the outbreak in the Philippines. Euro Surveill. 2014;19(15): pii=20773. Available online: http://www.eurosurveillance.org/ViewArticle.aspx?Articleld=20773

Article submitted on 14 April 2014 / published on 17 April 2014

\section{To the editor:}

We thank Mandal et al. for their letter in response to our paper. Firstly we would like to point out that most the concerns raised are due to the fact that our article was a preliminary report, which was also stated in the title. It was intended to rapidly inform about an outbreak of measles affecting European and non-European citizens that was ongoing at the time of publication and to alert public health, clinical and laboratory experts in various countries of the possibility of cases among people who had been on the cruise. In fact, most of those concerns are being addressed in the on-going investigation.

With regard to the potential source of the outbreak, we intentionally refrained from indicating that the ongoing measles cluster between 20 February 2014 and 1 March 2014 would unequivocally suggest a point-source outbreak with a unique primary case. Although this is a sensible hypothesis, we are currently analysing a large amount of data from all crew members employed on the ship between 2 January and 9 April 2014, to specifically address this question and other open issues. This also includes the identification of the primary case, the actual duration of the epidemic, the overall number of cases among the crew members and potential risk factors for infection. The long time period under investigation includes a long pre-epidemic period (about seven weeks before symptom onset of the earliest cases on 20 February) to identify any potentially unrecognised case, and a 32-day post-epidemic period (i.e. about twice the median incubation time after the last case recorded on board) to confirm the end of the outbreak. The results will be published once the data analysis has been completed.

In the microbiological results section we explained that "phylogenetic analysis demonstrated that identified sequences were $100 \%$ identical to each other, confirming a common origin, and to two British strains identified in February 2014 (MVs/Brighton.GBR/8.14/ and MVs/Tonbridge.GBR/7.14/, not shown)". In fact, we did not link the cruise outbreak ship to B3 strains indigenous from the United Kingdom (UK). In the letter Mandal et al. state that the outbreak strain (MVs/ Tonbridge.GBR/5.14) is identical to that identified in an English resident who had clearly been infected in the Philippines, and that our epidemiological and microbiological findings are consistent with the cruise ship outbreak being linked to the ongoing outbreak in the Philippines rather than to indigenous measles in the UK. This hypothesis is not contradicted by our preliminary analysis. However, at the time of our analysis, neither the measles nucleotide database (MeaNS) nor Blast showed sequences from the Philippines belonging to $B_{3}$ genotype. We did not have any evidence to conclude where the strain responsible for the cruise ship outbreak was initially acquired. In fact, the primary case could have been a passenger as well as a crew member who may have been infected anywhere (including the Philippines, the UK or Italy). The evidence is not convincing enough to draw a conclusion that the crew members from the Philippines were the source of the outbreak following direct importation from their home country.

Finally, concerning the attack rate among passengers, we should stress that the inference of $0.1 \%$ made by the authors of the letter is not supported by the available data, since we do not have information on the exact number of cases among passengers. It is worth noting that we will hardly be able to obtain the precise number of cases among passengers. In fact, as the duration of the cruise (seven days) is shorter than the incubation time of measles (7-18 days), no passenger is expected to develop symptoms while on board. Therefore passengers are not likely to take part in the diseases propagation within the ship, although this hypothesis could not be definitely ruled out. In addition, these cases will only occasionally be brought to our attention by local health authorities throughout the world. All these issues have been clearly discussed in our paper.

At present, activities are underway to better describe the epidemiological features of this outbreak and to provide final conclusions on the event. 
None declared.

Authors' contributions

All authors are part of the outbreak investigation team and reviewed and approved the manuscript.

\section{References}

1. Mandal S, Ramsay M, Brown K. Measles on a cruise ship: links with the outbreak in the Philippines. Euro Surveill. 2014;19(15): pii=20774.

2. Lanini S, Capobianchi MR, Puro V, Filia A, Del Manso M, Kärki

$T$, et al. Measles outbreak on a cruise ship in the western

Mediterranean, February 2014, preliminary report. Euro

Surveill. 2014;19(10): $\mathrm{pii}=\mathbf{2 0 7 3 5}$ 